\title{
Downregulation of Dicer, a component of the microRNA machinery, in bladder cancer
}

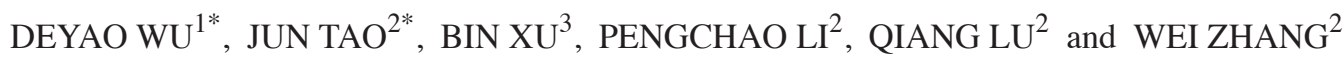 \\ ${ }^{1}$ Department of Urology, The Fourth Affiliated Hospital of Nantong Medical College, Yancheng City No. 1 \\ People's Hospital; ${ }^{2}$ Department of Urology, First Affiliated Hospital of Nanjing Medical University, Nanjing; \\ ${ }^{3}$ Department of Urology, Affiliated Zhongda Hospital of Southeast University, Nanjing, Jiangsu, P.R. China
}

Received September 17, 2011; Accepted December 12, 2011

DOI: $10.3892 / \mathrm{mmr} .2011 .711$

\begin{abstract}
Dysregulation of microRNA metabolism has been observed in a variety of human cancers. In this study, we evaluated the expression of the enzymes of the machinery Dicer, Drosha and DGCR8, in transitional cell carcinomas (TCCs) of the urinary bladder. The expression of Dicer, Drosha and DGCR8 was analyzed using semi-quantitative RT-PCR in clinical specimens from normal bladder mucosa, TCCs and their normal adjacent tissues (NATs). Immunohistochemistry was performed to compare the expression of Dicer in normal, TCCs and NATs. Our study demonstrated that Dicer mRNA levels in TCCs were significantly lower compared to normal samples and NAT samples. The immunohistochemistry results revealed that Dicer protein levels in TCCs were significantly downregulated compared to normal bladder mucosa and NATs. Our data demonstrated that Dicer is significantly downregulated in TCCs compared to paired NAT samples and normal samples, suggesting that reduced expression of Dicer may play an important role in bladder cancer.
\end{abstract}

\section{Introduction}

microRNAs (miRNAs) are minuscule RNA molecules approximately $22 \mathrm{nt}$ in length, and which regulate pathways that are essential to most biological processes, including differentiation, proliferation and apoptosis (1). Production and function of miRNAs require a set of proteins collectively referred to as the miRNA machinery. miRNAs encoded in the genome are transcribed by RNA polymerase II to primary transcripts, pri-miRNAs (2). The next step of miRNA maturation is the nuclear cleavage of pri-miRNA to a 60 - to 70 -nt stem-loop

Correspondence to: Professor Qiang Lu or Dr Wei Zhang, Department of Urology, First Affiliated Hospital of Nanjing Medical University, 300 Guangzhou Road, Nanjing, Jiangsu 210029, P.R. China

E-mail: luqiang0405@163.com

E-mail: zhangwei_njmu@126.com

${ }^{*}$ Contributed equally

Key words: bladder cancer, Dicer, microRNA machinery intermediate precursor, namely pre-miRNA. This step requires a 650-kDa microprocessor complex that comprises Drosha, an RNase III endonuclease, and its cofactor, DiGeorge syndrome chromosomal region 8 (DGCR8) (3). The nuclear RNase III Drosha catalyzes the first processing step together with the double-stranded RNA (dsRNA) binding protein DGCR8 generating pre-miRNAs. The next cleavage employs the cytoplasmic RNase III Dicer producing miRNA duplexes. Finally, Argonautes are recruited with miRNAs into an RNA-induced silencing complex (RISC) for mRNA recognition (4).

Dicer is a cytoplasmic RNase III type endonuclease, an essential protein component of the miRNA machinery with a key function in the generation of miRNAs and small interfering RNAs (siRNAs) (1). Its expression is upregulated in prostate cancers and precursor lesions of lung adenocarcinomas, but reduced in ovarian and lung cancers. Furthermore, both low and high levels of Dicer have been correlated with poor prognosis in cancer patients (5). Since Dicer catalyzes the biosynthesis of miRNAs and siRNAs which in turn regulate the expression of many genes, the expression of the Dicer gene itself may be a highly regulated process (6-8). Discrepancies in the dysregulation of Dicer expression among the various tumor types have been attributed to tissue-specific differences and/or to the degree of the aggressiveness of the cancer (9).

Drosha also belongs to the class of RNase III endonucleases. Drosha has two RNase III domains and one double-stranded RNA binding domain (dsRBD). The two RNase III domains (RIIIDa and RIIIDb) form an intramolecular dimer and cleave the $3^{\prime}$ and $5^{\prime}$ strands of the stem, respectively. Since the dsRBD of Drosha is insufficient for substrate binding, Drosha needs a partner protein surrogating the RNA recognition function. DGCR8 is the cofactor that interacts with Drosha and forms a functional complex called the 'Microprocessor' (10-12). The human DGCR8 gene is located on chromosome $22 \mathrm{q} 11$ and is expressed ubiquitously from fetus to adult (13). DGCR8 contains two dsRBDs and recognizes the unique features of the pri-miRNA, which include the single-stranded RNA (ssRNA) segments flanking a stem of appropriate length. DGCR8 anchors at the ssRNA-dsRNA junction and directs Drosha to cleave approximately 11 bp away from the junction (14). In an experiment where DGCR8 was depleted by RNAi, pri-miRNAs were found to accumulate, whereas pre-miRNA and mature miRNA levels decreased. Moreover, the addition 
Table I. Primer sequences for RT-PCR.

\begin{tabular}{lll}
\hline Gene & \multicolumn{1}{c}{ Forward $\left(5^{\prime} \rightarrow 3^{\prime}\right)$} & \multicolumn{1}{c}{ Reverse $\left(5^{\prime} \rightarrow 3^{\prime}\right)$} \\
\hline Dicer & GATGGTGGTCCACGAGTCACA & CATCAGGCAACTCTCGGGTTC \\
Drosha & GAGGATTAGCAACCTATCGGACTG & GCCCGTGAGCATACAGCATAAA \\
DGCR8 & TGAATGTGAGAACCCAAGTGAGC & CAGGGATGAGGATTTCCAGTGTAG \\
GADPH & GAAATCCCATCACCACTTCCAGG & GAGCCCCAGCCTTCTCCATG \\
\hline
\end{tabular}

of recombinant DGCR8 slightly reduced non-specific cleavage by Drosha.

In the present study, the expression of Dicer, Drosha and DGCR8 was analyzed using semi-quantitative RT-PCR and immunohistchemistry in clinical specimens from normal bladder mucosa, transitional cell carcinomas (TCCs) and their normal adjacent tissues (NATs). The mRNA levels of Dicer were found downregulated in the vast majority of TCCs as compared to normal tissues and NAT samples. According to the results of immunohistochemistry, the protein levels of Dicer were also lower in TCCs compared to normal samples and NATs. The expression of Drosha and DGCR8 was not significantly different between TCCs and their NAT samples.

\section{Materials and methods}

Patients and clinical specimens. Tissue specimens used in this study were collected at the Jiangsu Province Hospital, the first affiliated hospital with Nanjing Medical University. Tissues were obtained after patients provided informed written consent under a general tissue collection protocol approved by the Institutional Review Board. Tissues were snap-frozen in liquid nitrogen and stored at $-80^{\circ} \mathrm{C}$.

RNA extraction and semi-quantitative RT-PCR. Total RNA was extracted from tissue samples with TRIzol reagent (Invitrogen, Carlsbad, CA, USA) according to the manufacturer's instructions. The integrity and yield of RNA were confirmed by agarose gel electrophoresis and absorbance reading at $260 \mathrm{~nm}$. RNA was stored at $-80^{\circ} \mathrm{C}$ for subsequent experiments. cDNA synthesis was performed using the reverse transcription system (Toyobo). Primers are shown in Table I. The following PCR cycling parameters were employed: $95^{\circ} \mathrm{C}$ for 5 min, followed by 42 cycles at $95^{\circ} \mathrm{C}$ for $45 \mathrm{sec}, 56^{\circ} \mathrm{C}$ for $1 \mathrm{~min}, 72^{\circ} \mathrm{C}$ for $1 \mathrm{~min}$ and then $72^{\circ} \mathrm{C}$ for 7 min. Equal volumes of PCR products were resolved in agarose gel, visualized with ethidium bromide and photographed. Images were analyzed with AlphaImager 2200.

Immunohistochemical staining. Tissue samples were fixed in 4\% 3-heptanone in phosphate-buffered saline (PBS) for $24 \mathrm{~h}$ and embedded in paraffin. Tissue sections (5- $\mu \mathrm{m}$ thick) were prepared in polylysine-coated slides and all steps were performed at room temperature. Slides were initially heated at $56^{\circ} \mathrm{C}$ in an oven for $2 \mathrm{~h}$. After being dewaxed in xylene and rehydrated in gradient alcohols, the slides were boiled in $10 \mathrm{mmol} / \mathrm{l}$, sodium citrate buffer $\mathrm{pH} 6.8$ for $10 \mathrm{~min}$ in a microwave oven. After being treated with $3 \%$ hydrogen peroxide in methanol to inactivate the endogenous peroxidase activity, the samples were blocked with blocking solution (5\% goat serum in PBS) for 20 min and then incubated with anti-human Dicer antibodies. After incubation with the primary antibody overnight at $4^{\circ} \mathrm{C}$, secondary biotinylated antibodies and $\mathrm{ABC}$ reaction solution were applied sequentially according to commercial kit instructions. After counterstaining with haematoxylin and briefly washing with $30 \mathrm{mmol} / \mathrm{l}$ ammonium hydroxide, the slides were mounted with permanent mounting medium. Each set of experiments was conducted in triplicate and carried out under the same experimental conditions.

For quantitative analysis of immunostaining intensity, we computed the integrated optical density (IOD). Digitally fixed images were analyzed at x400 magnification using an AxioImager A1 light microscope equipped with Image Pro Plus 6.0. IOD was calculated for arbitrary areas, each sample analyzed with the same size. All data are expressed as mean values, and statistical analysis was applied to compare the results from the various experimental groups.

Statistical methods. Data are presented as the means $\pm \mathrm{SD}$ and compared using the Student's t-test in Stata software (version 8.2; StataCorp LP, College Station, TX, USA). P<0.05 was considered to denote statistical significance.

\section{Results}

Expression of Dicer, Drosha and DGCR8 mRNA in normal bladder mucosa, TCCs and NATs. First, we examined the relative mRNA levels of Dicer, Drosha and DGCR8 in normal, TCCs and NATs using semi-quantitative RT-PCR. We used 7 normal bladder tissue specimens and 20 paired samples of TCCs and NAT samples. The expression of Drosha and DGCR8 was not significantly different between TCCs and their NAT samples (data not shown). However, Dicer mRNA levels in TCCs were significantly lower compared to normal samples and NAT samples (Fig. 1A and B). We then compared the expression of Dicer in normal, TCCs and NATs by immunohistochemistry. For quantitative analysis of immunostaining intensity, we computed the IOD with Image Pro Plus 6.0. According to the results, Dicer protein levels in TCCs were significantly downregulated compared to normal and NAT samples (Fig. 2A and B). Dicer, but not DGCR8 or Drosha, was dysregulated in TCCs.

Relationship between Dicer mRNA levels and the clinicopathological features in patients with TCCs. Dicer mRNA expression levels are associated with clinicopathological features in ovarian carcinoma and lung cancer (15). Therefore, we examined whether the expression levels of Dicer are associated with tumor grade and stage. The statistical analysis 


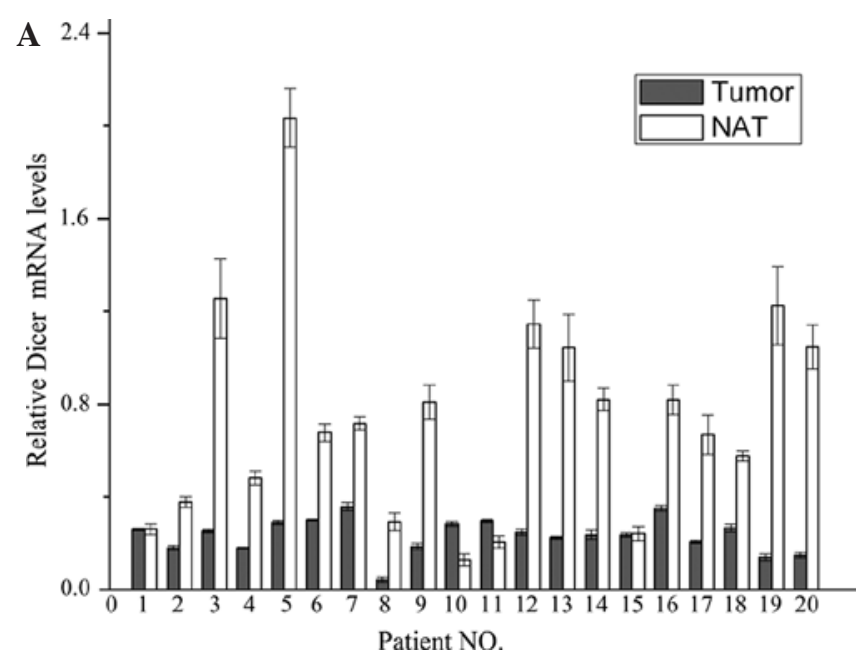

B

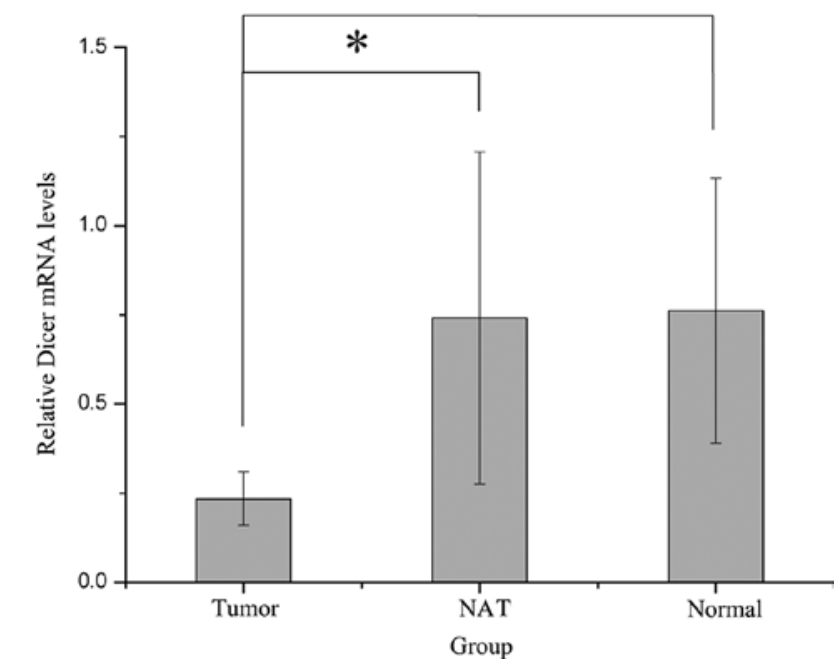

Figure 1. (A) Relative Dicer mRNA levels in paired bladder cancer tissues and adjacent non-cancerous bladder tissues (NATs). Signal intensities in pixels for Dicer were divided by the respective intensity of GADPH. The relative Dicer mRNA levels were significantly lower in tumor tissues compared to the NATs. (B) Average relative Dicer mRNA levels in bladder cancer, NATs and normal bladder mucosa. The average relative Dicer mRNA levels of TCCs were lower compared to the NATs and normal samples. ${ }^{\wedge} \mathrm{P}<0.05$ compared to normal samples. ${ }^{*} \mathrm{P}<0.05$ compared to NATs.

showed that there was no significant association between the Dicer expression levels and the tumor grade or stage (Fig. 3A and $\mathrm{B})$.

\section{Discussion}

In the present study, we evaluated the expression of Dicer, Drosha and DGCR8 in TCCs. We presented definitive evidence that in a significant fraction of bladder cancers the expression of Dicer was downregulated. To the best of our knowledge, this is the first report demonstrating alterations in the expression of Dicer in TCCs.

As an evolutionarily conserved gene pertaining to the function of the ancient RNAi pathway, Dicer is crucial to cell differentiation and proliferation. Dicer depletion by knockout is lethal to embryonic development for it is essential to stem cell proliferation and differentiation (16). Recently, dysregulation of Dicer in malignancies has been of great interest

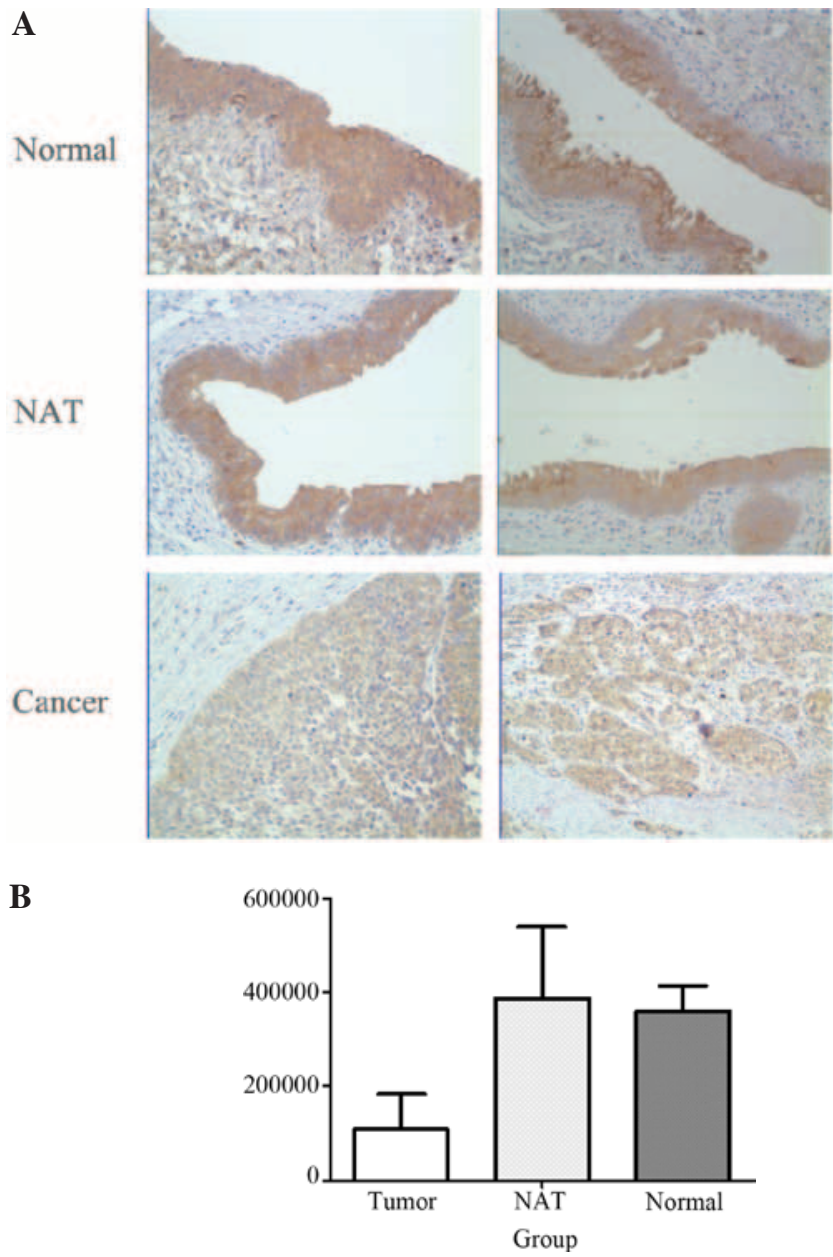

Figure 2. (A) Representative immunohistochemical staining for Dicer in normal samples, NATs and TCCs. Dicer protein levels in TCCs were significantly downregulated compared to normal samples and NATs. (B) IOD represents the immunostaining intensity of Dicer in normal samples, NATs and TCCs. The average Dicer protein levels of the bladder cancer group were lower compared to the NATs and normal samples.

in oncology. Studies have shown that Dicer is upregulated in prostate adenocarcinomas, ovarian serous carcinomas, esophageal cancers and acute myeloid leukemia (AML), and downregulated in hepatocellular carcinomas, lung cancers and ovarian carcinomas (17-19). The present study showed that Dicer was downregulated in TCCs compared to their NAT samples or normal bladder mucosae. These findings were in accordance with those of lung cancer. This may be explained by the similar molecular basis and risk factors between lung cancer and TCC, such as age, gender, smoking habits and exposure to carcinogens.

The downregulation of Dicer was associated with poor prognosis in ovarian carcinomas, while higher Dicer mRNA expression was a poor prognostic factor in esophageal cancers (1). However, Martin et al showed that Dicer levels are not associated with clinical outcomes in patients with AML (20). Wu et al found that there was no significant association between Dicer expression levels and clinical characteristics, including gender, age, tumor number, tumor size and distant metastasis (15). In the present study, we revealed that Dicer expression was not associated with clinicopathological features, such as tumor stage and grade. However, we found 

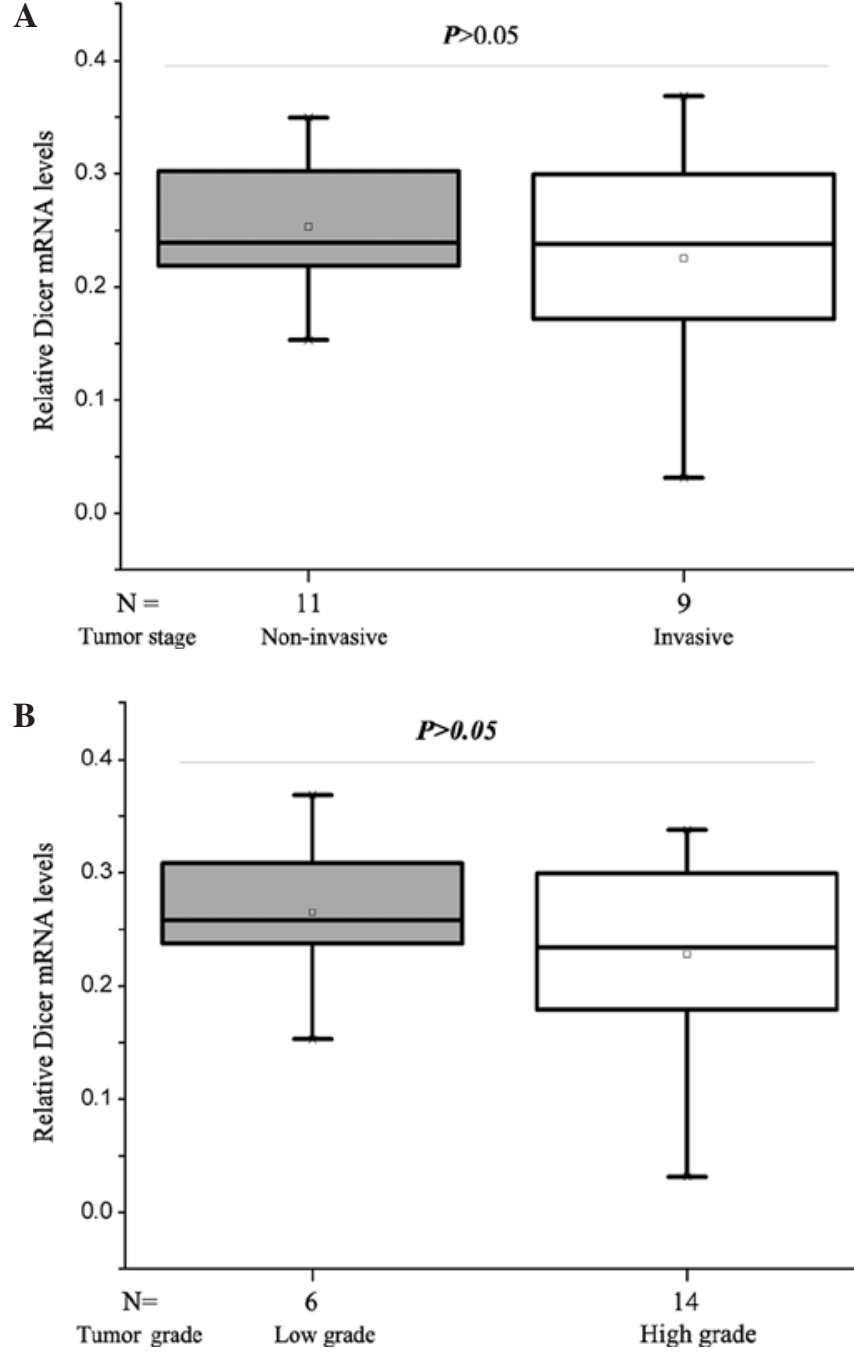

Figure 3. Relationship between Dicer mRNA levels and clinicopathological features in patients with bladder cancer. The relative Dicer mRNA levels of patients with bladder cancer have no significant correlation with (A) tumor stage and (B) tumor grade.

that the Dicer mRNA levels in non-invasive and low-grade TCCs were higher compared to invasive and high-grade TCCs, although the results did not reach statistical significance. The small amount of tissue samples may have been a contributing factor to the results. Thus, further studies with larger sample size are required to reach a conclusive result.

The underlying mechanism of Dicer downregulation was particularly interesting. Several mechanisms contribute to Dicer's dysregulation in human cancers, including genomic alterations, epigenetic modifications and alternative promoter usage. The human Dicer gene (Dicerl) is mapped to the subtelomeric region of chromosome 14 (14q32.13). An extensive search of publicly available databases for neoplasms associated with genetic abnormalities at the $14 \mathrm{q} 32.13$ locus yield a number of human malignancies (21-23). In lung cancer, a fraction of adenocarcinomas lost Dicer expression as a result of deletions within the Dicer locus (24). As for the mechanisms of downregulated Dicer expression in human bladder cancer, it is possible that Dicer downregulation is induced by a genomic instability at $14 \mathrm{q} 32.13$. Several reports have indicated a deletion in chromosome $14 \mathrm{q}$ in TCCs, especially in more invasive diseases. Micro-deletion in this subtelomeric region $14 \mathrm{q} 32$ may be a very early event in the pathogenesis of TCCs (25-28).

Pampalakis et al analyzed the DNA methylation sites of the Dicer gene using the $\mathrm{CpGplot} / \mathrm{CpGreport}$, and found that the $\mathrm{CpG}$ island spans the first exon of the gene at position -587 to +682 , with a $\mathrm{C}+\mathrm{G}$ content of $73.05 \%, 147 \mathrm{CpG}$ dinucleotides and an observed ratio of 0.93 , significantly higher than the expected ratio (1). The presence of this strong $\mathrm{CpG}$ island indicates that genomic DNA methylation may account for Dicer downregulation in human cancers, including TCCs.

In addition, alternative promoter usage may also take part in the regulation of Dicer expression, as shown in earlier studies in breast cancer (29). Therefore, it may also be the reason for the downregulation of Dicer in TCCs.

Previous findings indicate that miRNAs may have a crucial function in cancer initiation and progression (30). However, it is unclear whether the observed upregulation or downregulation of miRNA expression simply reflects malignant degeneration of the tumor or directly causes tumor initiation and progression. Changes in the stoichiometry of miRNA machinery components are thought to explain abnormal miRNA profiles in different cancer types (31). Therefore, it is possible that the downregulation of Dicer is a prerequisite to the abnormal miRNA observed in cancers (15). Thus, the decreased expression of Dicer may be a cause rather than a consequence of bladder cancer.

Dicer is essential for miRNA biogenesis. Loss of Dicer in mice disrupts embryonic stem cell differentiation and is lethal during early development. Low levels of Dicer mRNA also affect normal cellular development and differentiation. Disruption of Dicer promotes hepatocarcinogenesis (32). Additionally, Dicer downregulation is frequently correlated with an invasive phenotype in lung adenocarcinomas as reported by Chiosea et al (24). In their study, downregulation of Dicer was found in areas of invasion and advanced diseases. Furthermore, in breast cancer cells downregulation of Dicer by miR-103/107 promoted cell motility and invasiveness, but did no harm in cell proliferation in vitro (33). Together, although Dicer depletion is unendurable to most cell types, moderate Dicer downregulation may confer a more invasive phenotype to certain cancer cells. Thus, low Dicer expression in TCCs may be a predisposed condition to aggressive disease. Further studies are necessary to illuminate this hypothesis.

In conclusion, our data demonstrate that Dicer is significantly downregulated in TCCs compared to paired NAT and normal samples, suggesting that reduced expression of Dicer may play an important role during the process of bladder cancer.

\section{Acknowledgements}

This study was supported by the Program of the Key Medical Department of Jiangsu Province, the Department of General Surgery of the Jiangsu Province Hospital, and the Department of Urology of the Jiangsu Province Hospital.

\section{References}

1. Pampalakis G, Diamandis EP, Katsaros D and Sotiropoulou G: Down-regulation of dicer expression in ovarian cancer tissues. Clin Biochem 43: 324-327, 2010. 
2. Lee Y, Kim M, Han J, et al: MicroRNA genes are transcribed by RNA polymerase II. EMBO J 23: 4051-4060, 2004.

3. Croce $\mathrm{CM}$ and Calin GA: miRNAs, cancer, and stem cell division. Cell 122: 6-7, 2005.

4. Berdnik D, Fan AP, Potter CJ and Luo L: MicroRNA processing pathway regulates olfactory neuron morphogenesis. Curr Biol 18: $1754-1759,2008$

5. Jakymiw A, Patel RS, Deming N, et al: Overexpression of dicer as a result of reduced let-7 microRNA levels contributes to increased cell proliferation of oral cancer cells. Genes Chromosomes Cancer 49: 549-559, 2010.

6. Nelson P, Kiriakidou M, Sharma A, Maniataki E and Mourelatos Z: The microRNA world: small is mighty. Trends Biochem Sci 28: 534-540, 2003.

7. Tijsterman M and Plasterk RH: Dicers at RISC; the mechanism of RNAi. Cell 117: 1-3, 2004.

8. Carmell MA and Hannon GJ: RNase III enzymes and the initiation of gene silencing. Nat Struct Mol Biol 11: 214-218, 2004

9. Grelier G, Voirin N, Ay AS, et al: Prognostic value of Dicer expression in human breast cancers and association with the mesenchymal phenotype. Br J Cancer 101: 673-683, 2009.

10. Denli AM, Tops BB, Plasterk RH, Ketting RF and Hannon GJ: Processing of primary microRNAs by the Microprocessor complex. Nature 432: 231-235, 2004

11. Gregory RI, Yan KP, Amuthan G, et al: The Microprocessor complex mediates the genesis of microRNAs. Nature 432 235-240, 2004

12. Han J, Lee Y, Yeom KH, Kim YK, Jin H and Kim VN: The Drosha-DGCR8 complex in primary microRNA processing. Genes Dev 18: 3016-3027, 2004.

13. Shiohama A, Sasaki T, Noda S, Minoshima S and Shimizu N: Molecular cloning and expression analysis of a novel gene DGCR8 located in the DiGeorge syndrome chromosomal region. Biochem Biophys Res Commun 304: 184-190, 2003.

14. Han J, Lee Y, Yeom KH, et al: Molecular basis for the recognition of primary microRNAs by the Drosha-DGCR8 complex. Cell 125: 887-901, 2006.

15. Wu JF, Shen W, Liu NZ, et al: Down-regulation of Dicer in hepatocellular carcinoma. Med Oncol 28: 804-809, 2011.

16. Kanellopoulou C, Muljo SA, Kung AL, et al: Dicer-deficient mouse embryonic stem cells are defective in differentiation and centromeric silencing. Genes Dev 19: 489-501, 2005.

17. Chiosea S, Jelezcova E, Chandran U, et al: Up-regulation of dicer, a component of the microRNA machinery, in prostate adenocarcinoma. Am J Pathol 169: 1812-1820, 2006.

18. Flavin RJ, Smyth PC, Finn SP, et al: Altered eIF6 and Dicer expression is associated with clinicopathological features in ovarian serous carcinoma patients. Mod Pathol 21: 676-684, 2008 .
19. Zhang X, Cairns M, Rose B, et al: Alterations in miRNA processing and expression in pleomorphic adenomas of the salivary gland. Int J Cancer 124: 2855-2863, 2009.

20. Martin MG, Payton JE and Link DC: Dicer and outcomes in patients with acute myeloid leukemia (AML). Leuk Res 33: e127, 2009.

21. Ihara Y, Kato Y, Bando T, et al: Allelic imbalance of $14 \mathrm{q} 32$ in esophageal carcinoma. Cancer Genet Cytogenet 135: 177-181, 2002.

22. Shao J, Li Y, Wu Q, et al: High frequency loss of heterozygosity on the long arms of chromosomes 13 and 14 in nasopharyngeal carcinoma in Southern China. Chin Med J 115: 571-575, 2002.

23. Takamizawa J, Konishi H, Yanagisawa K, et al: Reduced expression of the let-7 microRNAs in human lung cancers in association with shortened postoperative survival. Cancer Res 64: 3753-3756, 2004.

24. Chiosea S, Jelezcova E, Chandran U, et al: Overexpression of Dicer in precursor lesions of lung adenocarcinoma. Cancer Res 67: 2345-2350, 2007.

25. Fornari D, Steven K, Hansen AB, et al: Under-representation of bladder transitional cell tumour $9 \mathrm{q}, 11 \mathrm{p}$ and $14 \mathrm{q} \mathrm{LOH}$ in urine and impact on molecular diagnosis. Anticancer Res 25: 4049-4052, 2005.

26. Bulashevska S, Szakacs O, Brors B, Eils R and Kovacs G: Pathways of urothelial cancer progression suggested by Bayesian network analysis of allelotyping data. Int J Cancer 110: 850-856, 2004.

27. Von Knobloch R, Brandt H and Hofmann R: Molecular serological diagnosis in transitional cell bladder cancer. Ann NY Acad Sci 1022: 70-75, 2004.

28. Saran KK, Gould D, Godec CJ and Verma RS: Genetics of bladder cancer. J Mol Med 74: 441-445, 1996.

29. Irvin-Wilson CV and Chaudhuri G: Alternative initiation and splicing in dicer gene expression in human breast cells. Breast Cancer Res 7: R563-R569, 2005.

30. Calin GA, Sevignani C, Dumitru CD, et al: Human microRNA genes are frequently located at fragile sites and genomic regions involved in cancers. Proc Natl Acad Sci USA 101: 2999-3004, 2004.

31. Sand M, Gambichler T, Skrygan M, et al: Expression levels of the microRNA processing enzymes Drosha and dicer in epithelial skin cancer. Cancer Invest 28: 649-653, 2010.

32. Sekine S, Ogawa R, Ito R, et al: Disruption of Dicerl induces dysregulated fetal gene expression and promotes hepatocarcinogenesis. Gastroenterology 136: 2304-2315.e1-4, 2009.

33. Martello G, Rosato A, Ferrari F, et al: A microRNA targeting dicer for metastasis control. Cell 141: 1195-1207, 2010. 